Research Article

\title{
On the Transmission of COVID-19 and Its Prevention and Control Management
}

\author{
Pinhong Zeng (1D \\ Faculty of Economics and Business Administration, Yibin University, Yibin 644000, China \\ Correspondence should be addressed to Pinhong Zeng; 2004114002@yibinu.edu.cn
}

Received 17 June 2021; Accepted 20 July 2021; Published 3 August 2021

Academic Editor: Guangdong Wu

Copyright (c) 2021 Pinhong Zeng. This is an open access article distributed under the Creative Commons Attribution License, which permits unrestricted use, distribution, and reproduction in any medium, provided the original work is properly cited.

\begin{abstract}
The spread of an epidemic is a typical public emergency and also one of the major problems that humans need to tackle in the $21^{\text {st }}$ century. Therefore, the research on the spread, prevention, and control of epidemics is quite an essential task. This paper first briefly described and analyzed the development of COVID-19 and then introduced the basic epidemic models and idealized the population in the epidemic area by dividing them into four categories (Classes $S, E, I$, and $R$ ). After that, it set the relevant parameters of the basic SEIR model and the modified one and worked out the relevant differential equations and iterative equations. According to the feature of the epidemic situation and the changes in the number of contacts in different units of time, the epidemic data were substituted into the iterative equations for data fitting with an $R$ Package. Then, analysis was performed on the epidemiological features such as the transmission time and epidemic peak and the epidemic trend was evaluated. Finally, sensitivity analysis was conducted on the parameters (government control and recovery rate), and the results showed that measures such as government restrictions on travel (reducing the contacts between virus carriers and susceptible persons) can effectively control the scale of the outbreak.
\end{abstract}

\section{Introduction}

At present, there are 64 major infectious diseases in the world, spreading in 82 countries and regions. In addition to the well-known influenza, measles and dengue fever are spreading in more than 20 countries and regions [1]. For many years, infectious diseases have been a major test to the medical and health systems in various countries in the world.

In 2019, a type of viral pneumonia appeared in many countries around the world, with the characteristics of "human-to-human transmission" [2]. The pathogen was officially named severe acute respiratory syndrome coronavirus 2 (SARS-CoV-2) and the pneumonia caused by the virus was named coronavirus disease 2019 (COVID-19) by the Coronaviridae Study Group (CSG) of the International Committee on Taxonomy of Viruses (ICTV). Since the first case of pneumonia of unknown cause was reported on December 8, 2019, a total of 115,653,459 cases and $25,718,823$ deaths have been reported worldwide as of March $6,2021[3]$.
Thanks to the active medical treatment and preventive and control measures, the epidemic situation in China was effectively contained and the economic and social order was being recovered in an orderly manner as early as April 2020. However, the epidemic was still spreading rapidly in countries and regions outside China, turning Europe and North America into the hardest-hit areas.

Since the outbreak of COVID-19, a number of studies have analyzed the development of the epidemic based on epidemic dynamics models and related data, interpreted the epidemic trends in different regions of China, and put forward recommendations for epidemic prevention and control $[4,5]$. In addition, Zhan et al. [6] established a virus transmission spectrum using COVID-19 data in Chinese cities and evaluated the development of the epidemic in cities in Italy and South Korea. Zhuang et al. [7] estimated the basic transmission rate of COVID-19 in Italy and South Korea using the maximum likelihood method and forecasted the epidemic situation at the early stage. Li et al. [8] estimated the basic infection rates before and after Korea's prevention and control measures and 
analyzed the epidemic situation in Italy. Zheng et al. [9] used the state transition matrix model to analyze the development of the epidemic in South Korea and Italy. There are also studies that estimated the basic infection coefficient of COVID-19 [10-13], but most of them were carried out before March 5, 2020, earlier than the time when the epidemic broke out on a global scale and turned into a pandemic.

To prevent and control COVID-19, different countries have adopted different measures, resulting in different development trends of the epidemic. For example, due to large-scale gatherings, the number of confirmed cases of COVID-19 increased significantly in South Korea in mid-to-late February [14], and later, thanks to active prevention and control measures, the epidemic was effectively controlled. As of April 4, 2020, there had been 10,237 confirmed cases in South Korea. Italy had fewer COVID-19 patients than South Korea at the beginning of March 2020; but after that, the number of new confirmed cases per day remained high, and as of April 4, 2020, the total number of confirmed cases had reached 124,632, much higher than that in South Korea. In addition, the United States, Spain, France, Germany, the United Kingdom, and other countries were also experiencing a surge in the number of confirmed cases. The pandemic situation was very severe.

The susceptible-infected-recovered (SIR) model is a classic model for studying epidemic dynamics. It was established by Kermack and McKendrick in 1927 using the dynamic method [15]. Yu et al. proposed an SIR model based on time-varying parameters and predicted the development of the epidemic with time-varying parameters [16]. Based on the traditional SEIR model, Geng et al. predicted the development of the novel coronavirus (2019-nCoV) pneumonia epidemic by adding new parameters such as the rate of infection in the incubation period and the rate of change of infected population and evaluated the role of relevant interventions [17-19]. Yan et al. [20] introduced a time-delay process to construct an infectious disease model based on a time-delay dynamic system on the basis of the traditional dynamic model to predict the epidemic situation and evaluate the effectiveness of prevention and control measures. Bai et al. [21] established a nonautonomous dynamic model to predict the development trend of the epidemic by adding isolated susceptible persons and isolated latent persons into the SEIR model and proposed sensitivity analysis on effective regeneration number to emphasize the effectiveness of tracking isolation in epidemic prevention and control.

Based on the SEIR model, this study established and modified a basic SEIR epidemic model and performed data fitting and sensitivity analysis using an $R$ Package. In order to realize the trend prediction of the epidemic, the prevention and control suggestions were put forward, and the feasibility of the measures was effectively evaluated, so as to provide a reference for the subsequent epidemic prevention and control.

\section{Transmission and Distribution of SARS-CoV-2}

2.1. Data Source. The data relating to COVID-19 in this paper were all extracted from the statistics published on the official website of the National Health Commission of the
People's Republic of China [22] from January 23 to March 14,2020 , including the numbers of (new) confirmed and (new) suspected cases, deaths, discharged patients, people under medical observation, and people discharged from medical observation nationwide every day, used to construct the SEIR model and analyze the epidemic situation. Considering the rapid spread of SARS-CoV-2, government agencies actively took effective preventive measures, putting the virus transmission into a controlled state. After effective quarantines, many community residents were restricted from going out, and few people traveled across cities and provinces, putting the virus transmission into a highly controlled state.

2.2. Data Analysis. From the map of epidemic outbreaks in China in Figure 1 (from the official website of China Centers for Disease Control and Prevention), it can be seen that Hubei Province is in the darkest color, showing that Hubei was the hardest-hit area of COVID-19 in China. The first case of pneumonia with an unknown cause was also found in Wuhan, Hubei. This provided important information for the traceability investigation of the virus and the quarantine and observation of close contacts.

Figure 2 is a pie chart based on the epidemic data of various provinces in China posted on the official website of the National Health Commission as of 24:00 on March 13, 2020. From Figure 2, it can be clearly seen that Hubei Province had the most confirmed cases, followed by Guangdong Province and Henan Province. Due to the strict isolation and travel restriction measures implemented in China, as of mid-March, all provinces except for Hubei have been cleared of COVID-19 cases.

Then, based on the case data published on the official website of the National Health Commission, a line chart was drawn. It can be seen from Figure 3, with the strong intervention of the government, that the new cases across China were on a decreasing trend. In particular, the number of new confirmed cases reached the peak on February 12 and then gradually decreased; the number of new suspected cases reached the peak on February 5, and after medical observation, it gradually decreased; the number of recovered cases continued to increase, and the number of deaths gradually decreased. Later, various data also showed that the outbreak was basically contained.

\section{Review of Common Epidemic Models}

The basic mathematical model for an epidemic is a mathematical model constructed based on the characteristics of the epidemic, used to study the transmission speed, spatial range, and transmission routes of the epidemic so as to effectively prevent and control the epidemic.

According to the conditions of different individuals, the total population in the epidemic area can generally be divided into the following four categories:

(1) The susceptible: denoted as the $S$ group, referring to the group of people who have not been infected in a 


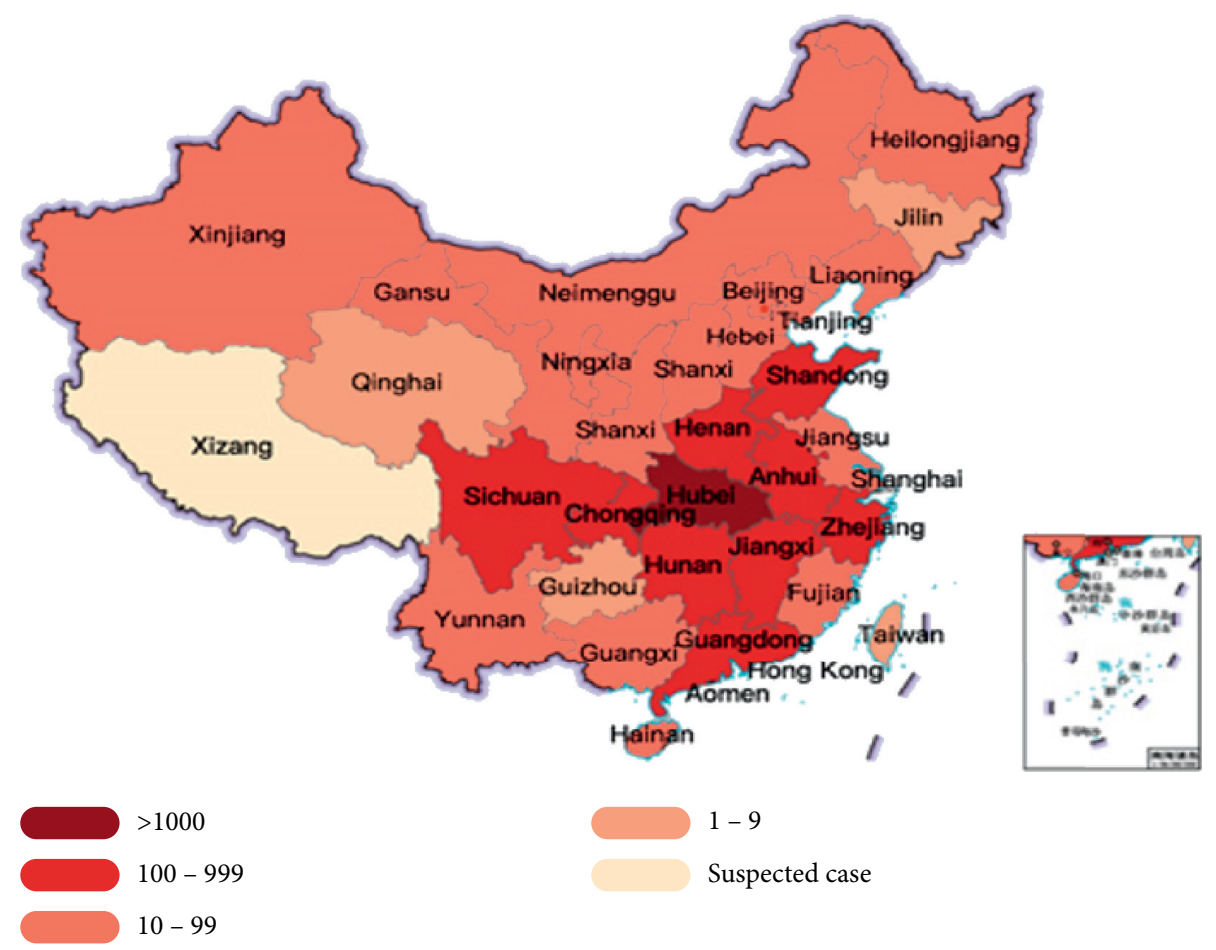

FIGURE 1: Epidemic outbreak map of China (as of 8:00 January 29, 2020).

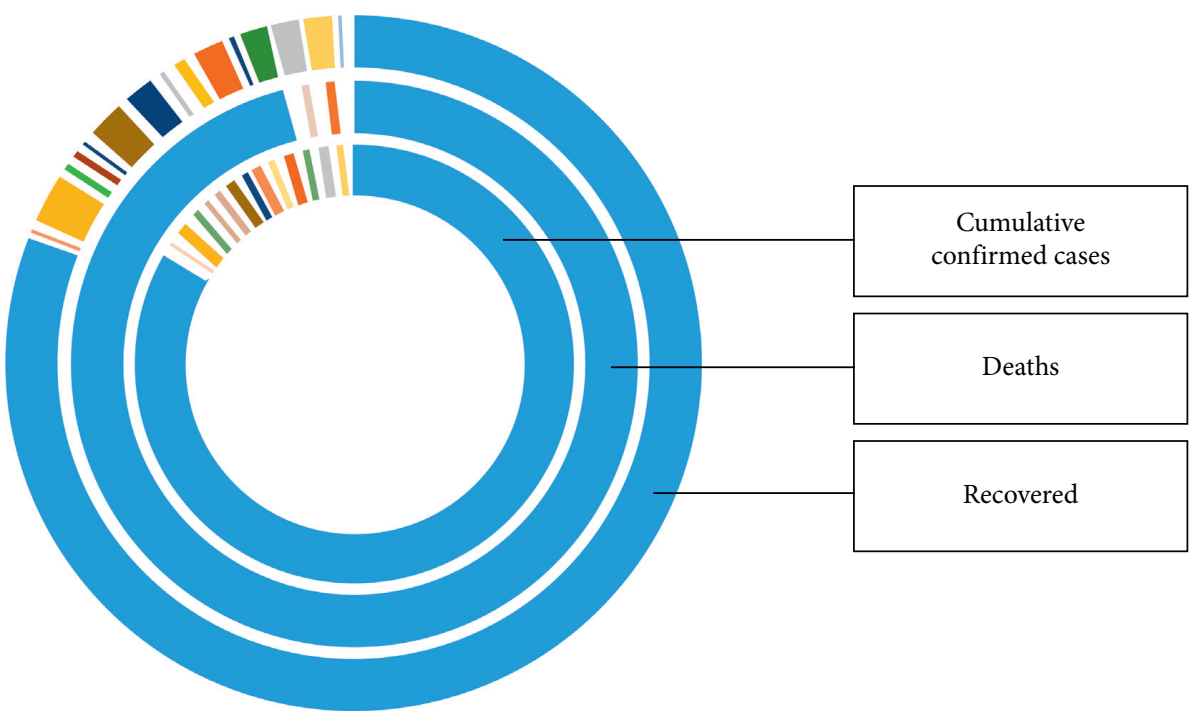
- Hubei
- Zhejiang
- Neimenggu
- Jiangxi
- Beijing
- Shandong
- Henan
- Fujian
Hong Kong
- Liaoning
- Hebel
- Jiangxi

- Guangdong

- Aomen

- Shanxi

- Taiwan

- Yunnan

- Chongqing

- Xinjiang

- Tianjing

- Gansu

- Xizang

- Hunan

- Heilongjiang

- Hainan

- Guizhou

- Qinghai

- Sichuan

- Jilin

- Guangxi

- Shanghai

- Jiangsu

- Ningxia

FIgURE 2: Distribution of COVID-19 cases by province. 


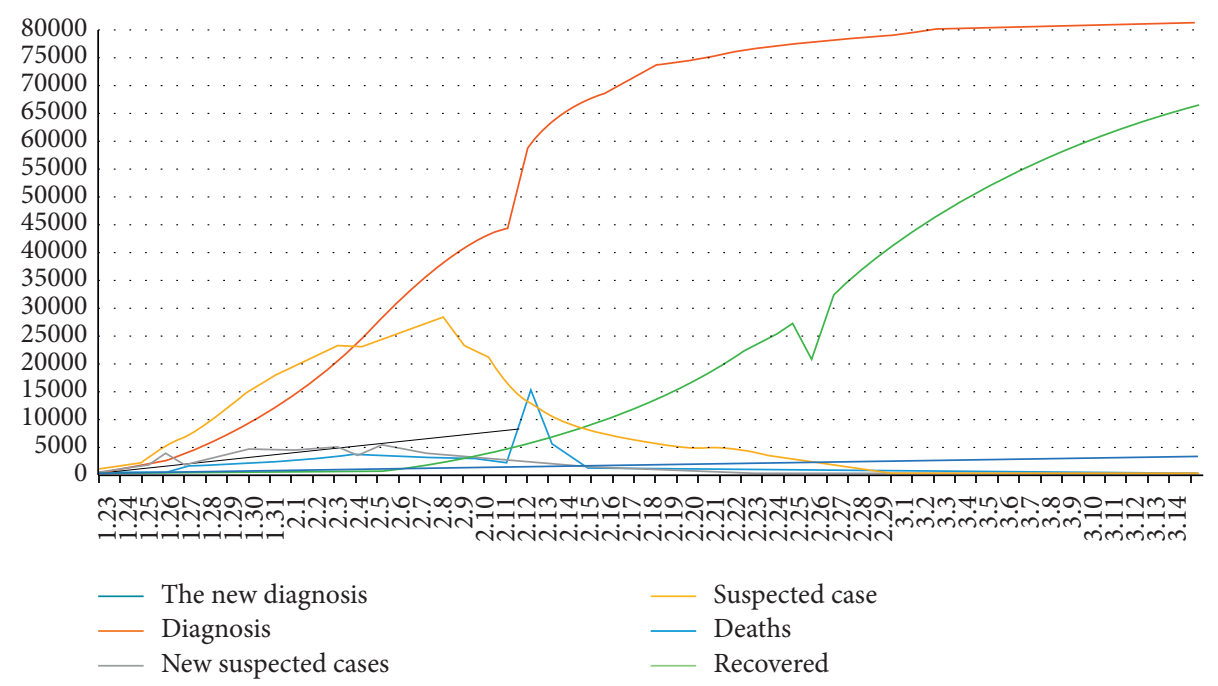

Figure 3: Line chart of COVID-19 cases (as of 24:00 March 14, 2020).

certain period of time but are susceptible to infection after contact with virus carriers.

(2) The exposed: denoted as the E group, referring to the group of people who have been in contact with the infectious and are in the latency stage of the disease. This is applicable to any infectious disease with a long latent period.

(3) The infectious: denoted as the $I$ group, referring to the group of people who have been infected with the infectious disease and can spread the disease to the susceptible, and turn them into the recovered or infectious.

(4) The recovered, denoted as the $R$ group, referring to the group of people who are cleared of the infectious disease and immune to the disease after being cured. If the immunity period is limited, the recovered will soon become the susceptible.

For the sake of intuitive and convenient research, Table 1 is a specific symbolic illustration diagram.

Suppose that the total population is $N$. During the outbreak of the epidemic, despite the prevention and control measures imposed by the government, there are still some individuals going out. Suppose the number of persons that each individual comes into contact with when going out is $C(t)$ and that the probability of such individual successfully spreading the virus to each contact is $\beta$, then there will be newly infected individuals. The birth rate and mortality rate of the population during the outbreak period are not taken into account in this study.

3.1. SI Model. The SI model is used for the situation in which the susceptible, after being infected with the disease, cannot be recovered. An example of such diseases is AIDS. The process is shown in Figure 4.

Suppose the population is divided into two groups- $S$ and $I$-and the total population is equal to the sum of the number of people in the $S$ group and the $I$ group; then, the following equations can be established for the number of newly infected cases and the number of healthy susceptible people reduced:

$$
\left\{\begin{array}{l}
\frac{\mathrm{d} S}{\mathrm{~d} t}=-\frac{C(t) \beta S I}{N}, \\
\frac{\mathrm{d} I}{\mathrm{~d} t}=\frac{C(t) \beta S I}{N} .
\end{array}\right.
$$

The original equations can be reorganized into the Bernoulli equations:

$$
\frac{\mathrm{d} I}{\mathrm{~d} t}-C(t) \beta I+\frac{C(t) \beta}{N} I^{2}=0 .
$$

And then there is

$$
I(t)=\frac{N I_{0}}{I_{0}+\left(N-I_{0}\right) e^{-C(t) \beta t}} .
$$

3.2. SIS Model. The SIS model is used for the situation in which individuals, after being infected with and recovered from an infectious disease, will return to susceptible individuals, who are likely to be infected again. An example of such diseases is influenza. The process is shown in Figure 5.

The equations are established as follows:

$$
\left\{\begin{array}{l}
\frac{\mathrm{d} S}{\mathrm{~d} t}=-\frac{C(t) \beta S I}{N}+\gamma I, \\
\frac{\mathrm{d} I}{\mathrm{~d} t}=\frac{C(t) \beta S I}{N}-\gamma I .
\end{array}\right.
$$

They can be simplified into

$$
\frac{\mathrm{d} I}{\mathrm{~d} t}-(C(t) \beta-\gamma) I+\frac{C(t) \beta}{N} I^{2}=0 .
$$

And it is solved as follows: 
TABLE 1: Description of symbols.

\begin{tabular}{lc}
\hline Symbol & Description \\
\hline $\begin{array}{l}\text { N } \\
(1 / \sigma)\end{array}$ & Total population \\
$\gamma$ & Infection rate (the probability of an exposed individual becoming an infected one) \\
$(1 / \gamma)$ & Average latent period \\
$(\beta S I / N)$ & Recovery rate \\
$\beta$ & Average duration of recovery (days) \\
$I(0)$ & Number of the infected \\
$E(0)$ & Transmission rate \\
$R(0)$ & Initially infected persons \\
$C(t)$ & Initial number of the exposed \\
$C\left(t_{0}\right)$ & Initial number of the recovered \\
$\alpha$ & Number of contacts per patient \\
$\beta_{2}$ & Initial number of contacts per patient \\
$C\left(t_{1}\right)$ & Recovery rate \\
\hline & Transmission rate of the exposed
\end{tabular}

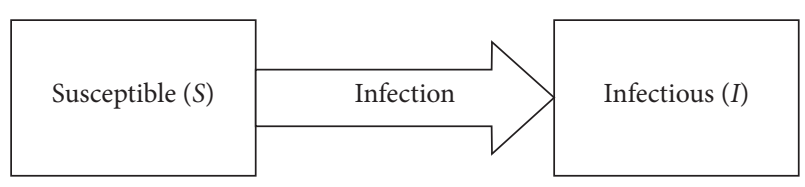

FIgURE 4: SI compartment chart.

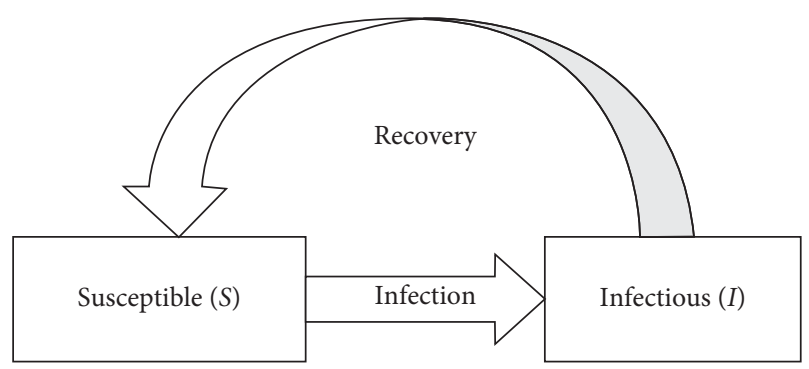

FIGURE 5: SIS compartment chart.

$$
I(t)=\frac{N I_{0}((C(t) \beta-\gamma) / C(t) \beta)}{I_{0}+\left(N((C(t) \beta-\gamma) / C(t) \beta)-I_{0}\right) e^{-(C(t) \beta-\gamma) t}} .
$$

3.3. SIR and SIRS Models. The SIR model is used for the situation in which individuals, after being infected with an acute infectious disease and recovered, will have antibodies and obtain permanent immunity. Examples of such diseases are smallpox and measles. The process is shown in Figure 6.

The equations are established as follows:

$$
\left\{\begin{array}{l}
\frac{\mathrm{d} S}{\mathrm{~d} t}=-\frac{C(t) \beta S I}{N}, \\
\frac{\mathrm{d} I}{\mathrm{~d} t}=\frac{C(t) \beta S I}{N}-\gamma I, \\
\frac{\mathrm{d} R}{\mathrm{~d} t}=\gamma I .
\end{array}\right.
$$

The SIRS model is used for the situation in which individuals, after being infected with an infectious disease and recovered, can be immune for only a limited period of time, and after that, they will become susceptible persons, with the risk of being infected again. The process is shown in Figure 7.

The equations are established as follows:

$$
\left\{\begin{array}{l}
\frac{\mathrm{d} S}{\mathrm{~d} t}=-\frac{C(t) \beta S I}{N}+\alpha R, \\
\frac{\mathrm{d} I}{\mathrm{~d} t}=\frac{C(t) \beta S I}{N}-\gamma I, \\
\frac{\mathrm{d} R}{\mathrm{~d} t}=\gamma I-\alpha R .
\end{array}\right.
$$

3.4. SEIR Model. In the SEIR model, the infected patients will experience a latent period in the beginning, meaning that after contacting with a virus carrier, they will not show symptoms until after a period of time, and by then, they become the carriers of the pathogen. After being recovered, they will have antibodies and not be infected again. Examples of such diseases are COVID-19, SARS, and so on. The process is shown in Figure 8.

The equations are established as follows:

$$
\left\{\begin{array}{l}
\frac{\mathrm{d} S}{\mathrm{~d} t}=-\frac{C(t) \beta S I}{N}, \\
\frac{\mathrm{d} E}{\mathrm{~d} t}=\frac{C(t) \beta S I}{N}-\sigma E, \\
\frac{\mathrm{d} I}{\mathrm{~d} t}=\sigma E-\gamma I, \\
\frac{\mathrm{d} R}{\mathrm{~d} t}=\gamma I .
\end{array}\right.
$$




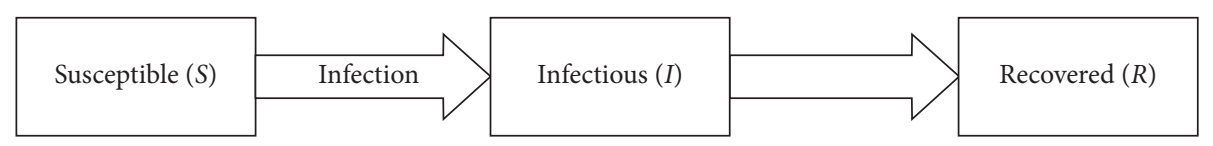

FIGURE 6: SIR compartment chart.

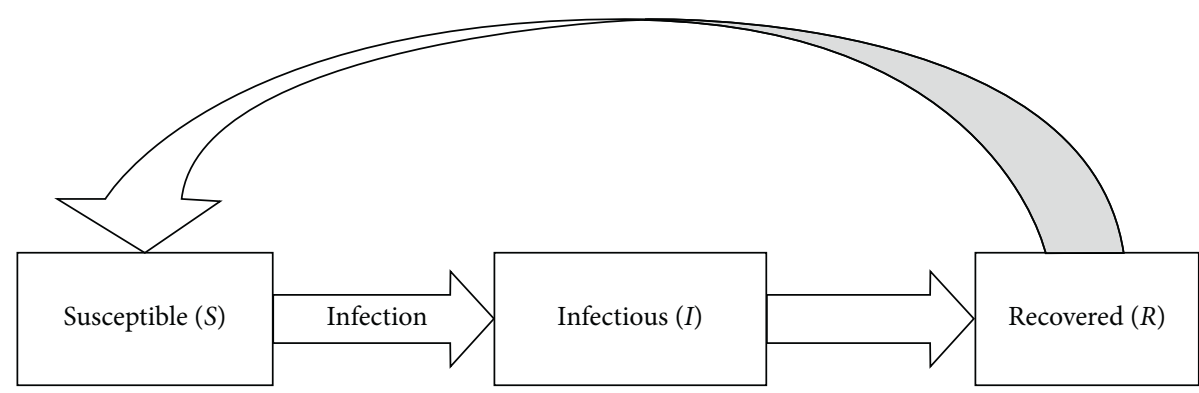

FIGURE 7: SIRS compartment chart.

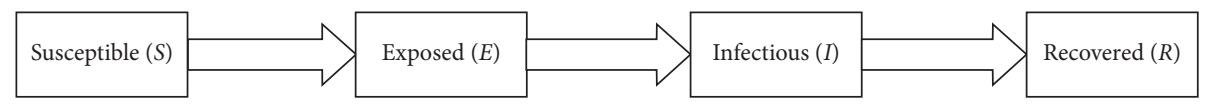

FIGURE 8: SEIR compartment chart.

From the above, it can be seen that it is actually a modified version based on the SIR model.

\section{Prediction of COVID-19 Development Based on the SEIR Model}

4.1. Basic Assumptions. COVID-19 has a latent period, so it is necessary to consider the individuals who have contacted the exposed. The following model assumptions are proposed for the transmission characteristics and changing trends of COVID-19 across China.

(1) This study is only intended to analyze and predict regional outbreaks within a short period of time, so it is assumed that the different groups of people are subject to conservation relations in the model, that is, $(t)+E(t)+I(t)+R(t)=$ constant. What is more, the data used are those obtained after all provinces across China activated the first-level lockdown, so there is no population flow. Also, the inflow, outflow, births, and deaths of the population are not taken into account.

(2) It is assumed that the initial number of infected persons is 1 .

(3) Individuals who have contacted the infected are put under medical observation for 14 days, during which time; they have no contact with the outside world.

(4) The individuals who are confirmed cases are completely quarantined and can no longer infect others.

(5) As mentioned above, if the COVID-19 patients, after being recovered, will no longer be isolated from others, the whole population can be divided into 4 groups of people, put in 4 independent compartments. The transforming relationships between them are shown in Figure 9.

In Figure 9, $(C(t) \beta I S / N)$ represents the rate at which the number of susceptible persons moves to the $E$ group per unit of time; $\sigma E$ is the number of people diagnosed with COVID19 per unit of time; and $\gamma I$ is the number of people recovered per unit of time. It should be noted that, based on our understanding of the actual situation, the exposed and infectious persons are both infectious. In the model, the patients refer to the individuals infected and hospitalized (including mobile cabin hospitals). The exposed, that is, the $E$ group, actually includes those in the latent period and those who have been infected but have not been discharged from the hospital.

\subsection{Parameters and Initial Value Setting.}

(1) Total population $N$ : the data of the total population was provided by the Hubei survey team of the $\mathrm{Na}$ tional Bureau of Statistics of The People's Republic of China. As of the end of 2019, the permanent population of Hubei Pro was 59.27 million.

(2) Infection rate $\sigma$ : according to the official website of the National Health Commission, based on the existing cases, the latent period of COVID-19 is about 7 days on average, but currently, the latent period has been extended. The duration of infection is $(1 / \sigma)$, so it can be calculated that $\sigma=0.143$.

(3) The determination of the expression of $C(t)$ [23].

(4) $\beta$ value: the value fitted by Xu et al. [24] based on data and the actual situation is adopted, that is, $\beta=0.074$. 


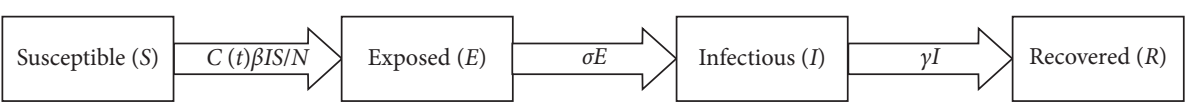

FIGURE 9: SEIR compartment chart.

\subsection{General Model and Analysis}

4.3.1. Modeling. According to the parameters, it can be found that $S / N$ represents the proportion of people who are not infected; $\beta I$ is the number of people who are contacted with and can be infected by patients, and $\gamma I$ is the number of people who have recovered. Then, based on the SEIR model, the daily increase in the number of people in each group can be expressed by the following equations:

$$
\left\{\begin{array}{l}
\frac{\mathrm{d} S}{\mathrm{~d} t}=-\frac{C(t) \beta I S}{N} \\
\frac{\mathrm{d} E}{\mathrm{~d} t}=\frac{C(t) \beta I S}{N}-\sigma E, \\
\frac{\mathrm{d} I}{\mathrm{~d} t}=\sigma E-\gamma I, \\
\frac{\mathrm{d} R}{\mathrm{~d} t}=\gamma I .
\end{array}\right.
$$

The iterative formulas can be obtained as follows:

$$
\left\{\begin{array}{l}
S_{n}=S_{n-1}-\frac{C(t) \beta I_{n-1} S_{n-1}}{N} \\
E_{n}=E_{n-1}+\frac{C(t) \beta I_{n-1} S_{n-1}}{N}-\sigma E_{n-1} \\
I_{n}=I_{n-1}+\sigma E_{n-1}-\gamma I_{n-1} \\
R_{n}=R_{n-1}+\gamma I_{n-1} .
\end{array}\right.
$$

The corresponding parameters are set as follows: the initial number of the infectious $I(0)=1$, that of the exposed $E(0)=0$, and that of the recovered $R(0)=0$.

4.3.2. Model Solution and Analysis. Without travel restrictions, assuming that in the range of $N=10000, C\left(t_{0}\right)=20$, the result calculated by an $R$ Package [25] is shown in Figure 10, where the inflection point of the epidemic appears at approximately two months and a half after the beginning of the outbreak. It can be seen that the numbers of the infectious and the exposed both increase first and then decrease and that the outbreak period is also relatively long. It can also be seen that the number of infected individuals does not conform to reality.

According to relevant reports, some exposed individuals who carry SARS-CoV-2 but do not show symptoms can still infect others. Therefore, the following improvements were made to the model.

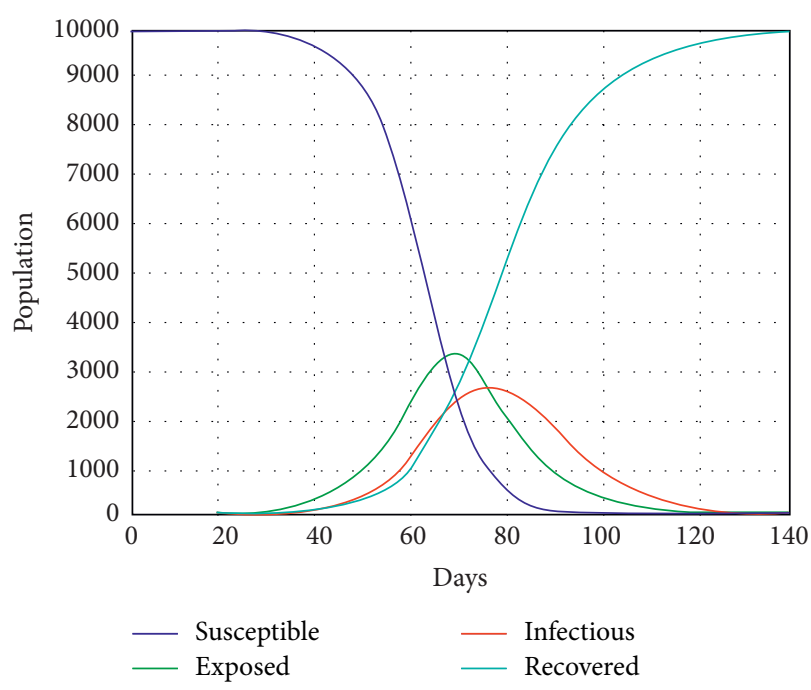

FIGURE 10: SEIR model fitting chart.

\subsection{Model Improvement and Analysis}

4.4.1. Modeling. The exposed and the infectious can both infect others. Suppose the contact rate among people is the same, and that the transmission rate is $\beta$, then the equations can be improved; that is, the daily increase in the number of people in each group can be expressed

$$
\left\{\begin{array}{l}
\frac{\mathrm{d} S}{\mathrm{~d} t}=-\frac{C(t) \beta S I}{N}-\frac{C\left(t_{1}\right) \beta_{2} E S}{N}, \\
\frac{\mathrm{d} E}{\mathrm{~d} t}=\frac{C(t) \beta S I}{N}-\sigma E+\frac{C\left(t_{1}\right) \beta_{2} E S}{N}, \\
\frac{\mathrm{d} I}{\mathrm{~d} t}=\sigma E-\gamma I, \\
\frac{\mathrm{d} R}{\mathrm{~d} t}=\gamma I .
\end{array}\right.
$$

The iterative formulas are as follows:

$$
\left\{\begin{array}{l}
S_{n}=S_{n-1}-\frac{C(t) \beta I_{n-1} S_{n-1}}{N}-\frac{C\left(t_{1}\right) \beta_{2} E_{n-1} S_{n-1}}{N}, \\
E_{n}=E_{n-1}+\frac{C(t) \beta I_{n-1} S_{n-1}}{N}-\sigma E_{n-1}+\frac{C\left(t_{1}\right) \beta_{2} E_{n-1} S_{n-1}}{N}, \\
I_{n}=I_{n-1}+\sigma E_{n-1}-\gamma I_{n-1}, \\
R_{n}=R_{n-1}+\gamma I_{n-1} .
\end{array}\right.
$$


According to the iterative formulas, the calculation results can be obtained.

4.4.2. Model Solution and Analysis. In the modified model, it can be seen from Figure 11 that the inflection point appears earlier, which, to a certain extent, verifies the feature that virus carriers can also infect others. This is also the reason why the epidemic situation is so difficult to contain.

\subsection{Parameter Sensitivity Analysis}

4.5.1. Sensitivity Analysis of $C\left(t_{0}\right)$. In 2.4 , through statistical analysis, it is determined that $C\left(t_{0}\right)=20$. Below is an analysis of the changes in the model results when $C\left(t_{0}\right)$ changes. This is to examine how a parameter affects the model results while other parameters remain unchanged. $C\left(t_{0}\right)$ represents the initial average number of contacts, which reflects the government's effort to control the epidemic.

(1) The Government Imposes Less Control. If the government imposes less control, the value of $C\left(t_{0}\right)$ will increase. Assuming that $C\left(t_{0}\right)=30$, it can be seen from Figure 12 that the number of the exposed has increased significantly, so in the same area, the healthy population are more likely to get infected.

(2) The Government Imposes More Control. If the government imposes more control, then the value of $C\left(t_{0}\right)$ will decrease. Assuming that $C\left(t_{0}\right)=10$, it can be seen from Figure 13 that the number of the exposed has decreased.

In the above two cases, the inflection point of the outbreak and the transmission period have changed, but not much. The above results also show that the government's travel restrictions can truly effectively control the spread of the epidemic by reducing the number of contacts.

4.5.2. Sensitivity Analysis of the Recovery Rate. It can be clearly seen from Figure 14 that if the recovery rate is increased to $\gamma=0.5$, the number of patients will be greatly reduced, the inflection point will appear earlier, and the transmission period will be relatively shortened. From this, it can be concluded that early detection, early isolation, early treatment, and at the same time improving the recovery rate of the infectious are very important to ending the outbreak as soon as possible.

\section{Prevention and Control Analysis}

From the previous SEIR epidemic model, it is known that both the exposed and the infectious can infect others. Since the contact rate among people is the same and the transmission rate is $\beta$, the key to preventing and controlling the epidemic is to minimize $\beta S(t)-\gamma$, by taking the following actions:

(1) Reduce $S(t)$ : Reduce the source of infection by reducing the number of the susceptible. The main

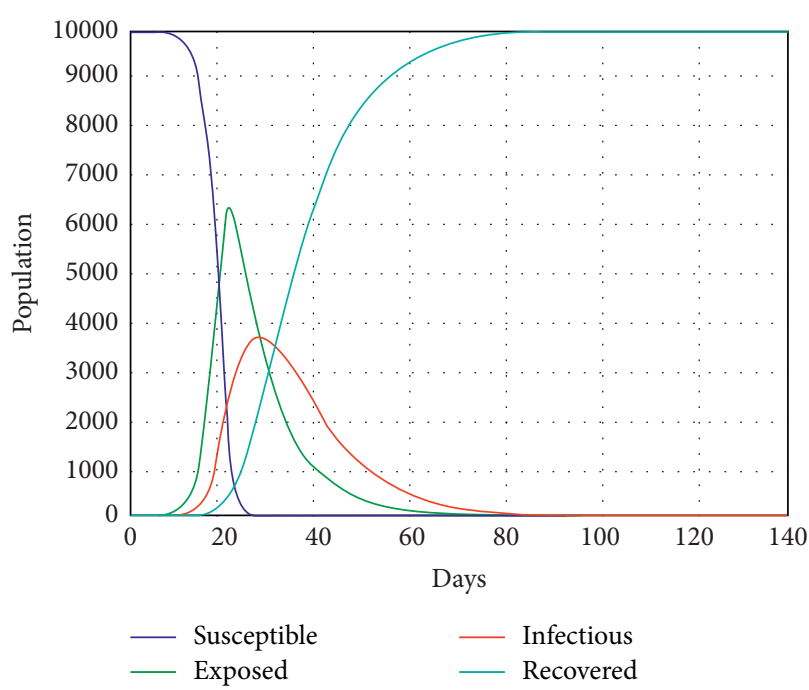

FIgURE 11: Fitting chart of the modified model.

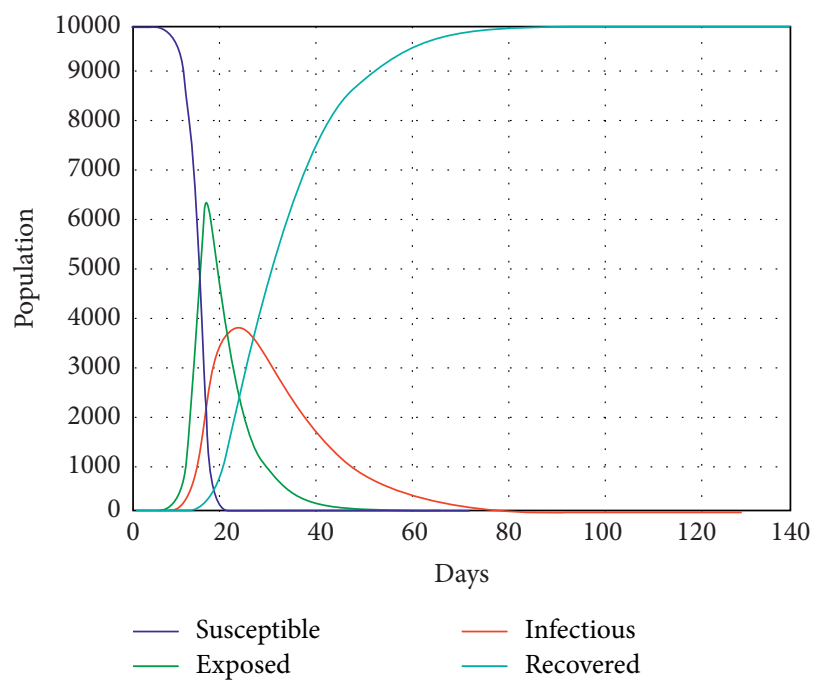

Figure 12: Fitting chart with more contacts per patient.

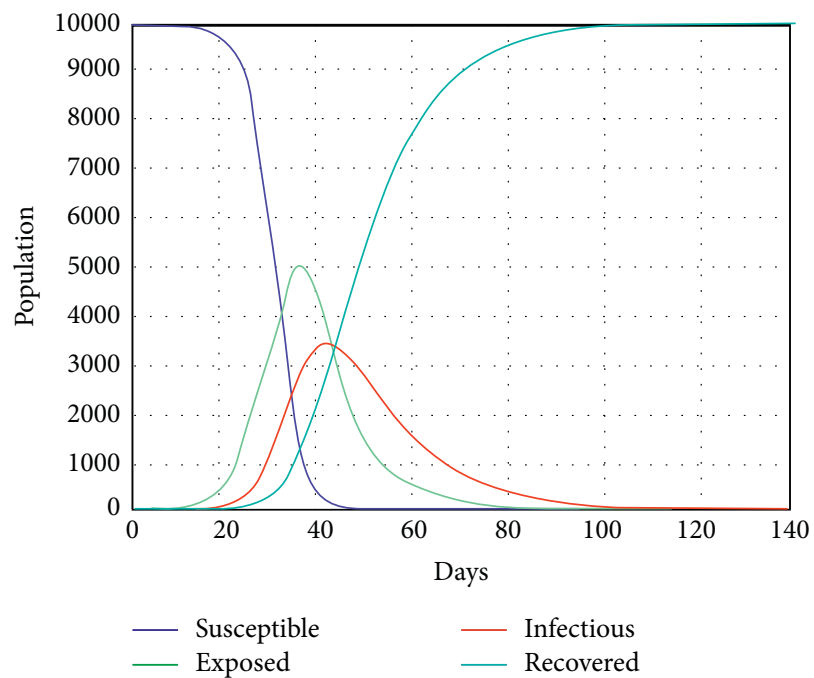

Figure 13: Fitting chart with fewer contacts per patient. 


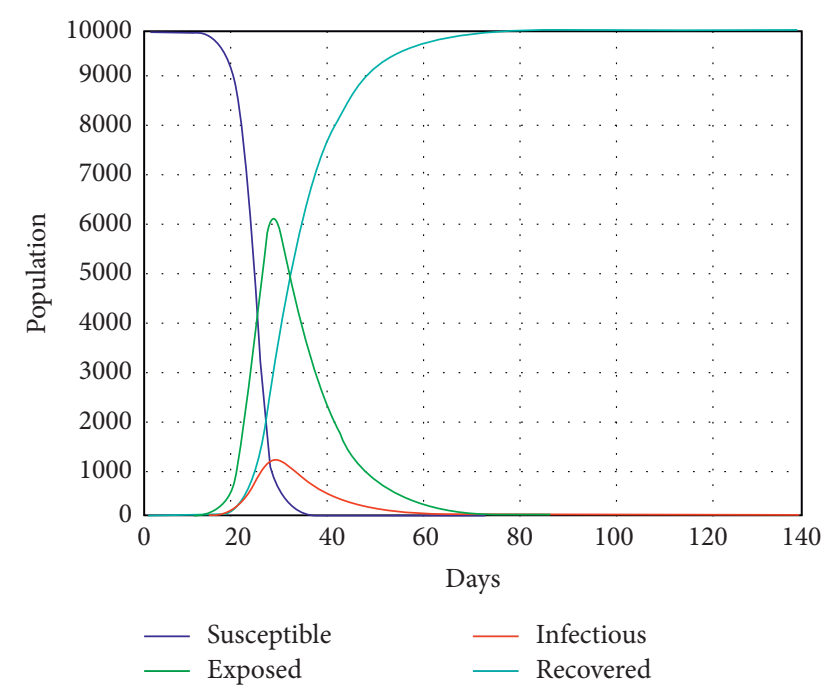

Figure 14: Fitting chart with a higher recovery rate.

methods are vaccination and isolation to prevent infection.

(2) Reduce $I(t)$ : Currently, patients are treated in isolation to reduce further infections.

(3) Reduce the infection rate $\beta$ : Further reduce individual contacts and improve sanitary conditions to block the spread of the virus. Common methods include frequent and scientific hand washing, wearing disposable gloves, wearing masks when going out, imposing community quarantine, and reducing public gatherings and shutdown of companies and schools.

(4) Increase the recovery rate $\gamma$ : speed up treatment, increase the recovery rate, and increase the immune population. Common measures include actively looking for specific therapeutic drugs, developing vaccines, enhancing physical fitness, and improving individual immunity. At the government level, actions to take include simplifying the drug approval process and speeding up drug development and clinical trials.

The above analysis shows that, before the vaccine is successfully developed, the traditional isolation method is indeed one of the most effective ways to prevent and control the epidemic. However, specifically, different isolation methods should be applied to different areas. For high-risk areas, such as Wuhan, absolute isolation is required, while for nonrisk susceptible areas, real-time protective isolation is required, such as isolation of streets, communities, and villages without COVID-19 cases. In addition, for different people, different methods should also be applied, like the isolation of susceptible individuals, isolation of contacts, and quarantine of suspected patients.

For the infectious group in the model, all suspected patients should be received and treated as much as possible. Those with fever and close contacts should be isolated, reported, diagnosed, quarantined, and treated as early as possible. Just as President $\mathrm{Xi}$ emphasized during the investigation on the prevention and control of COVID-19, the Chinese must fight and win the battle against the epidemic by mobilizing all resources and blocking the spread of the virus.

\section{Conclusions}

This paper used the basic SEIR epidemic model and then obtained the results with an $R$ Package using the data to describe the transmission pattern and development process of COVID-19. The main work done for this paper included acquiring the epidemic data from the official website, plotting relevant charts, performing data analysis, and understanding the relevant situation. In this outbreak, it was found that Hubei Province was the hardest-hit area. Then, the basic epidemic models were described, including the SI model, the SIS model, the SIR (SIRS) model, and the SEIR model. The SEIR model was given the greatest attention and applied to the outbreak of COVID-19. Differential equations and iterative equations were established, and with these equations, the final results were obtained. After comparison of the calculated results against the reality and considering the special feature of this epidemic, the equations were modified and calculations were performed again. The results obtained were more consistent with the reality. Sensitivity analysis was also performed on two parameters. Through comparison of the results and based on the actual situation, prevention and control recommendations were proposed.

\section{Data Availability}

The data used to support the findings of this study are available from the author upon request.

\section{Conflicts of Interest}

The author declares no conflicts of interest.

\section{References}

[1] H. Han, B. Wu, Y. J. Song, and J. J. Jia, "Outline of global infectious diseases outbreaks in december 2019," Disease Surveillance, vol. 35, no. 1, pp. 3-5, 2020.

[2] J. F.-W. Chan, S. Yuan, K.-H. Kok et al., "A familial cluster of pneumonia associated with the 2019 novel coronavirus indicating person-to-person transmission: a study of a family cluster," The Lancet, vol. 395, no. 10223, pp. 514-523, 2020.

[3] C. L. Li, W. T. Wu, and Z. Y. Pan, "Control effect analysis of new coronavirus pneumonia in Zhejiang province," Zhejiang Medical, vol. 42, no. 4, pp. 311-314, 2020.

[4] WHO, WHO Coronavirus Disease (COVID-19) Dashboard [EB/OL], World Health Organization, Geneva, Switzerland, 2021, http://lcovid19.who.int.

[5] X. Pan, "Quantitative analysis and prediction of the worldwide COVID-19 pandemic," IOP Conference Series: Earth and Environmental Science, vol. 693, no. 1, Article ID 012086, 2021.

[6] C. Zhan, C. K. Tse, Z. Lai, T. Hao, and J. Su, "Prediction of COVID-19 spreading profiles in Republic of Korea, Italy and Iran by data-driven coding," PLoS One, vol. 15, no. 7, Article ID e0234763, 2020. 
[7] Z. Zhuang, S. Zhao, Q. Lin et al., "Preliminary estimates of the reproduction number of the coronavirus disease (COVID-19) outbreak in Republic of Korea and Italy by 5 March 2020," International Journal of Infectious Diseases, vol. 95, pp. 308-310, 2020.

[8] L. Li, Z. Yang, Z. Dang et al., "Propagation analysis and prediction of the COVID-19," Infectious Disease Modelling, vol. 5, pp. 282-292, 2020.

[9] Z. Zheng, K. Wu, Z. Yao, X. Zheng, J. Zheng, and J. Chen, "The prediction for development of COVID-19 in global major epidemic areas through empirical trends in China by utilizing state transition matrix model," BMC Infectious Diseases, vol. 20, no. 1, pp. 710-712, 2020.

[10] Q. Li, X. Guan, P. Wu et al., "Early transmission dynamics in Wuhan, China, of novel coronavirus-infected pneumonia," New England Journal of Medicine, vol. 382, no. 13, pp. 1199-1207, 2020.

[11] J. Chen, L. Wu, J. Zhang et al., "Deep learning-based model for detecting 2019 novel coronavirus pneumonia on high-resolution computed tomography," Scientific Reports, vol. 10, no. 1, pp. 19196-19211, 2020.

[12] J. T. Wu, K. Leung, and G. M. Leung, "Nowcasting and forecasting the potential domestic and international spread of the 2019-nCoV outbreak originating in Wuhan, China: a modelling study," The Lancet, vol. 395, no. 10225, pp. 689-697, 2020.

[13] W. Zhou, Y. Xiao, and J. M. Heffernan, "Optimal media reporting intensity on mitigating spread of an emerging infectious disease," PLoS One, vol. 14, no. 3, Article ID e0213898, 2019.

[14] R. Takele, "Stochastic modelling for predicting COVID-19 prevalence in East Africa countries," Infectious Disease Modelling, vol. 5, pp. 598-607, 2020.

[15] W. O. Kermack and A. G. McKendrick, "A contribution to the mathematical theory of epidemics," Proceedings of the Royal Society of London. Series A, Containing Papers of a Mathematical and Physical Character, vol. 115, no. 772, pp. 700-721, 1927.

[16] Z. Yu, G. Zhang, Q. Liu, and Z. Lv, "Evaluation and prediction of COVID-19 epidemic based on time-varying parameter-SIR model," Journal of University of Electronic Science and Technology of China, vol. 49, no. 3, pp. 357-361, 2020.

[17] H. Geng, A. Xu, X. Wang et al., "The role of COVID-19 in the prevention and treatment of infectious diseases," Journal of Jinan University (Natural Science \& Medical Edition), vol. 41, no. 2, pp. 175-180, 2020.

[18] D. Gao and S. Ruan, "An SIS patch model with variable transmission coefficients," Mathematical Biosciences, vol. 232, no. 2, pp. 110-115, 2011.

[19] W. Wang and X.-Q. Zhao, "An epidemic model in a patchy environment," Mathematical Biosciences, vol. 190, no. 1, pp. 97-112, 2004.

[20] Y. Yan, Y. Chen, K. Liu, X. Luo, and J. Cheng, "Modeling and prediction of COVID-19 epidemic based on a class of timedelay dynamic systems," Science China: Mathematics, vol. 50, no. 3, pp. 385-392, 2020.

[21] N. Bai, C. Song, and R. Xu, "A study on COVID-19 epidemic prediction and control strategy based on dynamic model," Acta Mathematicae Applicatae Sinica, vol. 43, no. 3, pp. 483-493, 2020.

[22] National Health Committee of the People's Republic of China, Update on epidemic situation of novel coronavirus-infected pneumonia by 24:00 on Feb 4, 2020.
[23] H. C. Li, J. X. Li, W. J. Jin, L. S. Shi, and M. R. Duan, "Epidemic analysis of novel coronavirus (COVID-19) in Henan Province," Journal of Chongqing University of Technology, vol. 34, no. 4, pp. 59-65, 2020.

[24] J. N. Xu, R. Ma, Z. R. Zhao, and H. Cheng, "Preliminary prediction of the epidemic tendency of 2019 novel coronavirus pneumonia in Shandong province," Journal of Chongqing Normal University, vol. 37, no. 2, pp. 101-106, 2020.

[25] S. M. Jenness, S. M. Goodreau, and M. Morris, "Epimodel: an $\mathrm{R}$ package for mathematical modeling of infectious disease over networks," Journal of Statistical Software, vol. 84, no. 8, Article ID 29731699, 2018. 